\title{
A New Method of PV Array Faults Diagnosis in Smart Grid
}

\author{
Ze Cheng, Yucui Wang, and Silu Cheng \\ School of Electrical Engineering and Automation, Tianjin University, Tianjin 300072, China \\ Correspondence should be addressed to Ze Cheng; chengze@tju.edu.cn
}

Received 19 November 2013; Accepted 24 June 2014; Published 10 July 2014

Academic Editor: H. D. Chiang

Copyright (C) 2014 Ze Cheng et al. This is an open access article distributed under the Creative Commons Attribution License, which permits unrestricted use, distribution, and reproduction in any medium, provided the original work is properly cited.

\begin{abstract}
A new fault diagnosis method is proposed for PV arrays with SP connection in this study, the advantages of which are that it would minimize the number of sensors needed and that the accuracy and anti-interference ability are improved with the introduction of fuzzy group decision-making theory. We considered five "decision makers" contributing to the diagnosis of PV array faults, including voltage, current, environmental temperature, panel temperature, and solar illumination. The accuracy and reliability of the proposed method were verified experimentally, and the possible factors contributing to diagnosis deviation were analyzed, based on which solutions were suggested to reduce or eliminate errors in aspects of hardware and software.
\end{abstract}

\section{Introduction}

A stable and reliable operation of the photovoltaic (PV) arrays is desirable for better performance and prolonged lifetime of the PV systems. However, PV arrays are highly susceptible to a variety of problems, such as hot spots, aging, and damage $[1,2]$, which could significantly reduce the power output or even permanently damage the batteries $[1,3]$. It is thus of paramount importance to detect and locate these faults in PV arrays.

Fault diagnosis methods for PV arrays can be broadly classified into those based on infrared images and those based on electrical signals. The former method makes use of the inherent property of the infrared images that there is a clear temperature difference between the defective and nondefective PV arrays $[2,4]$. However, it has been criticized for being inaccurate, use of expensive and delicate instruments, and delayed reaction. In recent years, considerable effort has been devoted to upgrading the hardware and software but results in no significant improvement in the fault diagnosis of largescale PV arrays. On the other hand, the electrical method, despite its limitations such as use of large number of sensors, low accuracy, inadaptability to large-scale PV arrays, and vulnerability to environmental influences, has found a place in fault diagnosis. An electrical method proposed by Japanese scholars applied the high frequency reaction measurement with time domain analysis for the detection of failed modules $[5,6]$, which had no real-time property and a low realistic possibility of operation. Despite these problems, most of the fault diagnosis methods based on voltage or current sensors can detect and locate certain kinds of faults [5, 7-11].

In a previous study, a new PV connection was designed to detect the faults of large-scale PV systems, in which a large number of sensors were embedded and "data fusion" technique was used [7]. Another approach was to use a switching matrix to connect the solar adaptive bank to the solar PV module branches [8]. Some parameters of PV module, such as shunt resistance, series resistance, and diode factor, have been shown to be closely related to PV array faults [9]. A novel method was then proposed to acquire the $I-V$ curves of $\mathrm{PV}$ modules strings, and the failures were indicated by the variations of the parameters based on the $I$ - $V$ curves. Two methods, capacitance measurement (ECM) and time-domain reflectometry (TDR), were presented to locate the faults in the PV module strings [5]. ECM could detect the disconnection position in the string without the effects of irradiance change, while TDR could detect the degradation position (series resistance increase) by the change of response waveform. However, these techniques still have the limitations described previously. In addition, existing fault diagnosis functions for PV inverter can only provide fault information in the branch.

In this study, a new sensor-embedded method is proposed for the detection of PV array faults, which has better integrated practical value. This method not only reduces the number of sensors needed to collect the necessary data 
and the cost of the whole system but also improves the accuracy and anti-interference ability with the introduction of fuzzy group decision-making theory [12-17]. Fuzzy group decision-making theory has been applied to fault diagnosis of rotating and intelligent instrument $[13,14]$ and proved to be especially suitable for group decision-making problems with different forms of preference information and incomplete certain information on weights. The final goal of group decision making is to find the best solution among a set of feasible alternatives, which can best reflect the preferences of the group of decision makers as a whole. In this study, we consider five "decision makers" contributing to the diagnosis of PV array faults, including voltage, current, environmental temperature, panel temperature, and solar illumination. The proposed method is experimentally verified and factors that cause diagnosis deviation are analyzed; then solutions are suggested to reduce or eliminate errors in aspects of hardware structure and software design [18].

\section{A New Diagnosis Method for PV Array Faults}

\subsection{PV Array Connection Structure and Sensor Detection Structure}

2.1.1. PV Array Connection Structure. Each PV cell can produce only a limited voltage and current. To increase voltage and current output, it is desirable to connect individual cells in series, parallel, series-parallel (SP), or total cross tied (TCT) to form larger arrays [19]. It needs to consider the effect of connection structure and detection mode of voltage and current sensors in monitoring large-scale PV arrays. A variety of detection structures have been proposed based on different connection structures. For example, some sensors were embedded in PV arrays with TCT connection. However, these structures tend to be complicated and costly.

2.1.2. A New Sensor Detection Structure. Detection structure preferably has the following characteristics: (1) using as few sensors as possible, (2) high resolution, and (3) being adaptable to large-scale PV arrays. A detection structure that complies with the above requirements is proposed in this study, as shown in Figure 1.

This detection structure is based on a $4 \times 8 \mathrm{PV}$ array with SP connection, where each symbol represents a solar panel, and there are three sensors (one current sensor and two voltage sensors) embedded in each branch. Thus if one solar panel fails, fault will be confined to the two adjacent panels.

If $I_{i}<I_{j}(0<i \leq 4,0<j \leq 4$ and $j \neq i)$, fault occurs in the $i$-th branch. Then the failed panel can be located according to the voltages measured by the two voltage sensors. There are four possibilities (PV panels are numbered from top to bottom).

(1) If No.1 or No.2 panel fails, then $V_{i 1}<V_{j 1}, V_{i 2}>V_{j 2}$, where $1 \leq j \leq 4$ and $j \neq i$;

(2) If No.3 or No.4 panel fails, then $V_{i 1}<V_{j 1}, V_{i 2}<V_{j 2}$, where $1 \leq j \leq 4$ and $j \neq i$;
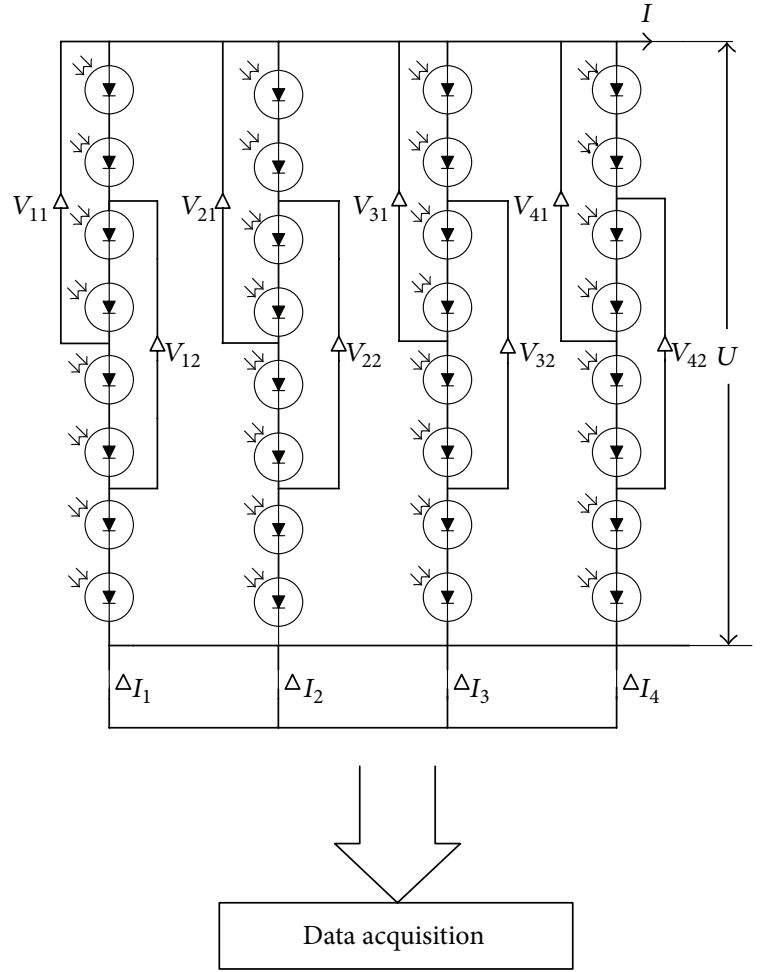

FIGURE 1: A new fault detection structure.

(3) If No.5 or No.6 panel fails, then $V_{i 1}>V_{j 1}, V_{i 2}<V_{j 2}$, where $1 \leq j \leq 4$ and $j \neq i$;

(4) If No.7 or No.8 panel fails, then $V_{i 1}>V_{j 1}, V_{i 2}>V_{j 2}$, where $1 \leq j \leq 4$ and $j \neq i$;

For the detection structure of $M \times N$ PV array ( $N$ branches, $M$ solar panels in each branch) shown in Figure 2, the resolution of fault location is assumed to be $L$ (accordingly, one voltage sensor is responsible for $2 \times L$ solar panels) and each branch has $p$ voltage sensors. Fault will be located based on the voltage and current data collected by a microcontroller.

(a) If

$$
\begin{aligned}
& V_{h r}<V_{i j}(0<i \leq N, i \neq h, 0<j \leq p) \\
& V_{h s}>V_{i j}(0<s \leq p, s \neq r, 0<i \leq N, i \neq h, 0<j \leq \\
& p) \\
& V_{i j}=V_{u v},(0<i, j, u, v \leq N ; i, j, u, v \neq h),
\end{aligned}
$$

fault occurs in No.h branch due to different sensor readings in this branch. Then it can be determined that the failed panel is within the range of the $r$-th sensor.

(b) If

$$
\begin{aligned}
& V_{h r}<V_{i j}, V_{h(r+1)}<V_{i j}(0<i \leq N, i \neq h, 0<j \leq p) \\
& V_{h s}>V_{i j}(0<s \leq p, s \neq r, s \neq r+1,0<i \leq \\
& N, i \neq h, 0<j \leq p) \\
& V_{i j}=V_{u v},(0<i, j, u, v \leq N ; i, j, u, v \neq h),
\end{aligned}
$$

fault occurs in No.h branch due to different sensor readings in this branch. Then it can be determined that the failed panel is within the cross range of No.r and No. $(r+1)$ sensor. 


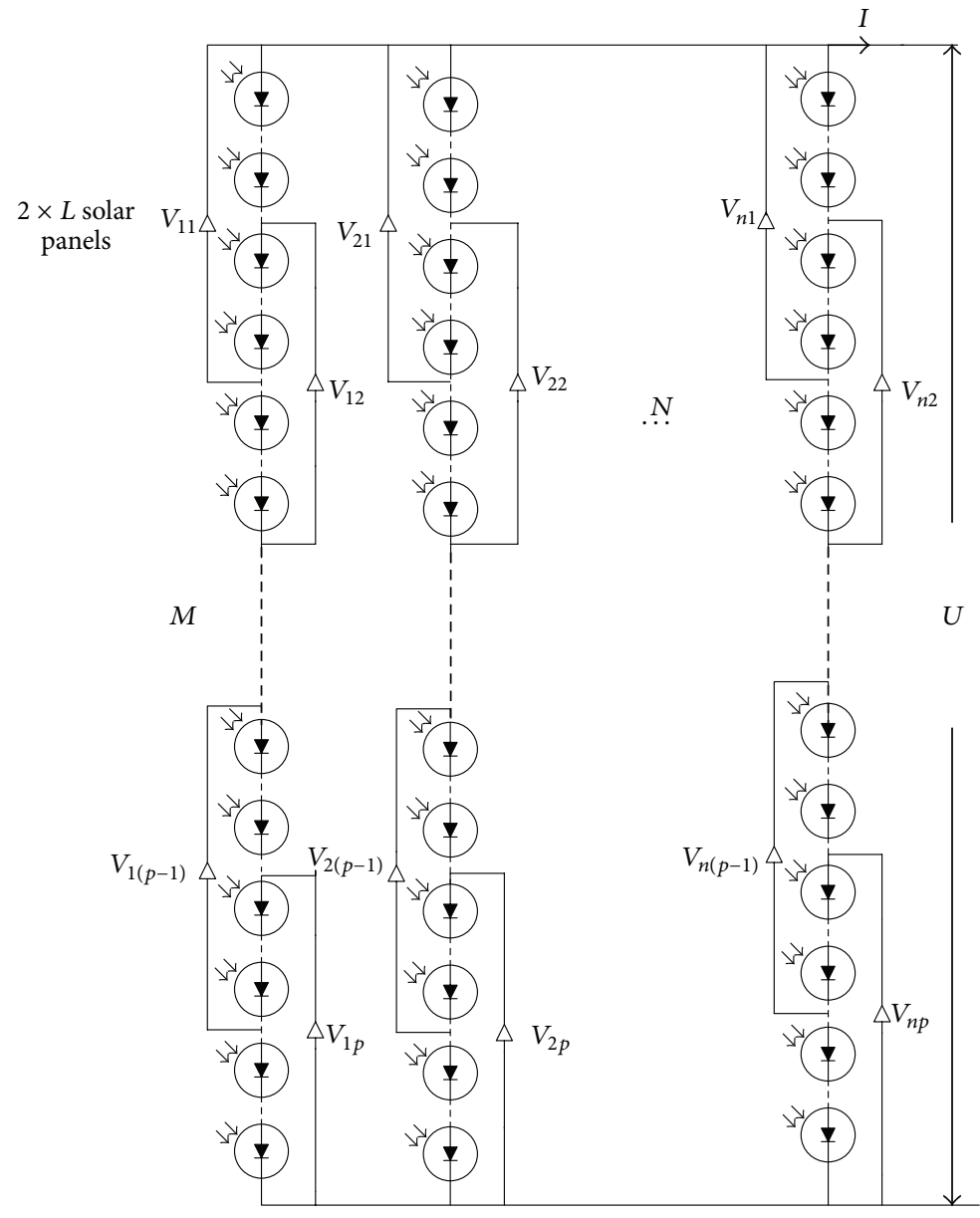

FIgURE 2: $M \times N$ detection structure.

As described in Figure 2, fault location is determined by a process of logical deduction. The detection structure proposed in this study considers the cross range of voltage sensors, thereby minimizing the number of sensors needed and eventually the cost of the system. This would be particularly obvious with the increase of $M$.

The relationship between $X$ (the number of sensors needed), $L, M$, and $N$ is

$$
X=\left[\frac{M}{3 \times L}\right] \times 2 \times N+N,
$$

where $[M / 3 \times L]$ is to eliminate the decimal part.

Equation (1) shows that $X$ is inversely proportional to $L$. Thus, the higher the accuracy of fault positioning, the larger the number of sensors needed.

\subsection{Fuzzy Group Decision-Making Theory in the Diagnosis of $P V$ Array Faults}

2.2.1. Fuzzy Fault Diagnosis. Uncertainty is a universal characteristic of decision-making problems. As we will see, it is particularly relevant to the diagnosis of PV array faults due to the dynamic nature and uncertainty-contingency and fuzziness-of the detection signals. A key premise underlying fuzziness is that there appears to be no clear-cut difference between two phenomena. It is necessary to establish the relationship between fuzzy problems and inherent factors in a mathematical way, and the result can be obtained by the fuzzy mathematics [20]. Given different attributes of PV fault diagnosis system and uncertainties in data processing, fuzzy method is applied in this study to process the measurement data and evaluate the fault level.

2.2.2. Group Decision-Making Theory in Fault Diagnosis. Group decision making is an important topic in system management. The primary purpose of group decision making is to find the most preferred solution among a set of feasible alternatives provided by multiple decision makers, which can best reflect the preferences of the group of decision makers as a whole and therefore avoid decision mistakes to a maximum extent [21].

PV array faults could cause changes in voltage, current, and panel temperature, and abnormalities in these parameters are, in turn, indicative of PV array faults. Although no direct relationship has been established between PV array faults and environmental temperature or solar irradiance, both of them are introduced as decision makers in the diagnosis of PV array faults, as shown in Figure 3.

Let $D=d_{1}, d_{2}, d_{3}, \ldots, d_{m}$ be a set of decision makers, $O=o_{1}, o_{2}, o_{3}, \ldots, o_{m}$ a set of alternatives, and $\lambda=$ 


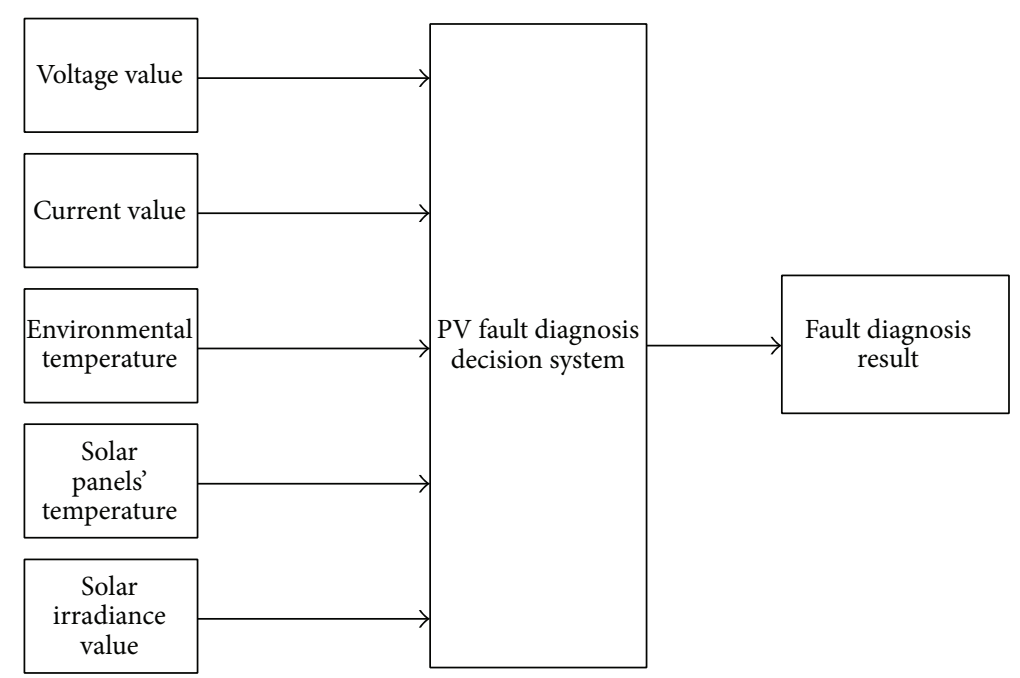

FIGURE 3: Group decision-making system for PV array faults.

$\left[\lambda_{1}, \lambda_{2}, \lambda_{3}, \ldots, \lambda_{m}\right]$ the weight vector of decision makers, respectively. The alternatives that No.i decision maker offers are $W^{(i)}=\left[W_{1}^{(i)}, W_{2}^{(i)}, W_{3}^{(i)}, \ldots, W_{n}^{(i)}\right]$. For any given $W^{(i)}$, the rank vector $R^{(i)}=\left[r_{1}^{(i)}, r_{2}^{(i)}, r_{3}^{(i)}, \ldots, r_{n}^{(i)}\right]$ can be calculated, where $r_{j}^{(i)}$ is the rank of No.j alternative for No. $i$ decision member $\left(1 \leq r_{j}^{(i)} \leq n\right)$. $W^{(i)}$ is graded according to the hierarchical fuzzy quantitative analysis before calculating $R^{(i)}$. The minimum unit value depends on the features of both decision makers and fault diagnosis.

(1) The generalized distance of decision makers is

$$
d(i, j)=\gamma_{i j}+\theta_{i j} \cdot i
$$

where

$$
\begin{gathered}
\gamma_{i j}=\frac{1}{n} \sum_{k=1}^{n}\left|r_{k}^{(i)}-r_{k}^{(j)}\right|, \\
\theta_{i j}=\arccos \left(\frac{W^{(i)} \cdot W^{(j)}}{\left\|W^{(i)}\right\| \cdot\left\|W^{(j)}\right\|}\right) .
\end{gathered}
$$

$\gamma_{i j}$ and $\theta_{i j}$ represent the degree to which the two decision makers are consistent.

(2) For any $d(i, j)=\gamma_{i j}+\theta_{i j} \cdot i$, the standard generalized distance is

$$
Q_{i j}=\frac{\gamma_{i j}}{\gamma_{\max }} \cdot \alpha+\frac{\theta_{i j}}{\theta_{\max }} \cdot \beta,
$$

where $\theta_{\max }=90$,

$$
\gamma_{\max }= \begin{cases}n / 2, & n \text { is even } \\ n / 2-1 / 2 n, & n \text { is odd }\end{cases}
$$

$\alpha$ is the rank weight coefficient of two weight vectors and $\beta$ is the angle weight coefficient that meet $\alpha+\beta=$ 1 and $\alpha>\beta$.
(3) Let $\gamma_{A}+\theta_{A} \cdot i$ be the remarkable consistency threshold and let $\gamma_{D}+\theta_{D} \cdot i$ be the serious divergence threshold, the values of which depend on the composition of decision makers and the attributes of decisionmaking problem. In the diagnosis of PV array faults, they would be determined by measurement data and experience. $Q_{A}$ and $Q_{D}$ are corresponding standard generalized distances.

The decision function of remarkable consistency is

$$
\varphi(i, j)= \begin{cases}1, & Q_{i j} \leq Q_{A} \\ 0, & Q_{i j}>Q_{A}\end{cases}
$$

The decision function of serious divergence is

$$
\psi(i, j)= \begin{cases}1, & Q_{i j} \geq Q_{D} \\ 0, & Q_{i j}<Q_{D}\end{cases}
$$

(4) GC and GD are the judgment matrix of the remarkable consistency and serious divergence, respectively:

$$
\begin{gathered}
\mathbf{G C}=\left\{c_{i j}\right\}_{m \times m} c_{i j}= \begin{cases}\varphi(i, j), & i \neq j, \\
1, & i=j,\end{cases} \\
\mathbf{G D}=\left\{x_{i j}\right\}_{m \times m} x_{i j}= \begin{cases}\psi(i, j), & i \neq j, \\
0, & i=j .\end{cases}
\end{gathered}
$$

(5) The consistency index is

$$
\mathrm{IAI}^{(i)}=\sum_{\substack{j=1 \\ j \neq i}}^{m} \frac{\varphi(i, j)}{m-1} .
$$

The divergence index is

$$
\mathrm{IDI}^{(i)}=\sum_{\substack{j=1 \\ j \neq i}}^{m} \frac{\psi(i, j)}{m-1} .
$$


(6) The proportion of decision makers that provide remarkably consistent opinions is

$$
\mathrm{GAI}=\sum_{i=1}^{m} \frac{\mathrm{IAI}^{(i)}}{m}
$$

The proportion of decision makers that provide seriously divergent opinions is

$$
\mathrm{GDI}=\sum_{i=1}^{m} \frac{\mathrm{IDI}^{(i)}}{m} .
$$

There are five decision makers $(m=5)$, including voltage, current, environmental temperature, panel temperature, and solar irradiance, denoted by $d_{V}, d_{I}, d_{\mathrm{TP}}, d_{\mathrm{TE}}$, and $d_{G}$, respectively, and five alternatives $(n=5, O=\{\mathrm{VL}, L, M, H, \mathrm{VH}\})$, including very low, low, medium, high, and very high fault probability. The standard generalized distance between two decision makers is

$$
\begin{aligned}
Q_{i j}= & \frac{\sum_{k=1}^{5}\left|r_{k}^{(i)}-r_{k}^{(j)}\right|}{12} \cdot \alpha \\
& +\frac{\arccos \left(\left(W^{(i)} \cdot W^{(j)}\right) /\left(\left\|W^{(i)}\right\| \cdot\left\|W^{(j)}\right\|\right)\right)}{90} \cdot \beta,
\end{aligned}
$$

where $i, j=d_{V}, d_{I}, d_{\mathrm{TP}}, d_{\mathrm{TE}}, d_{G}$. According to $Q_{A}$ and $Q_{D}$, the index can be calculated and final fault diagnosis can be made.

\section{Experiment and Analysis}

3.1. Experiment Design and Data Analysis. The proposed method is then verified experimentally with custom-made PV panels, as shown in Figure 4. The terminals of each PV monomer are independent so that they can be connected arbitrarily. It consists of four branches numbered from 1 to 4 . Temperature is measured by a DS18B20 digital thermometer and solar irradiance by a TSL230B light to frequency converter from TI company.

The data collected in this study are shown in Table 1 , where $V_{11}$ to $V_{42}$ are voltages, $I_{1}$ to $I_{4}$ are currents, $T_{e 1}$ to $T_{e 4}$ are environmental temperatures, $T_{p 1}$ to $T_{p 4}$ are panel temperatures, and $G_{1}$ to $G_{4}$ are solar irradiances, respectively.

Because neither environmental temperature nor solar irradiance has a direct effect on PV array faults, a special treatment is adopted. When they are normal, all the preference data are set to be 0.2 and the rank results to be in accordance with $r_{k}^{(I)}$ or $r_{k}^{(V)}$. However, when they are abnormal, the failure probability is reduced, VL and Lare increased, and $M, H$, and $\mathrm{VH}$ are decreased. Fuzzy quantitative analysis is performed with the other three decision makers using cross triangular membership function. The preference data of No.1 branch are shown in Table 2.

The rank results are shown in Table 3. Then the standard generalized distance $Q_{i j}$ can be calculated using (13), and the results are shown in Table 4 , where $\alpha=0.7, \beta=0.3, Q_{A}=$ 0.05 , and $Q_{D}=0.5$.

The evaluation indexes in Table 5 show that the overall consistency index is 0.20 and the divergence index is 0 ,

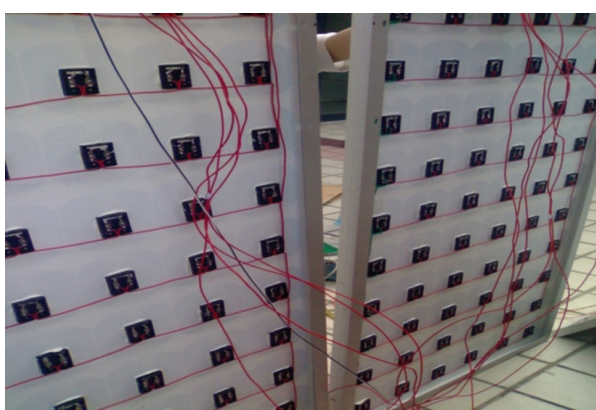

FIGURE 4: Custom-made solar panels.

indicating a high consistency between decision makers. Therefore, No.1 branch has a relatively high probability of faults. According to the judgment process described above, No.1 or No.2 PV cell in the first branch might fail.

By following the same process as above, we found that No. 2 and No. 3 branch have no fault, but No. 4 branch has fault. The remarkable consistency is 0.10 and the serious divergence is 0.60 , indicating a false fault detection. The deviation of the voltage and current from normal range may be due to environmental factors. A miscarriage of justice would happen if the decision is made on the basis of incomplete measurement data rather on group decision making in which a group of decision makers work collectively to find the best candidate from a set of alternatives.

3.2. Errors and Solutions. The precision of the system would decrease due to the errors inherent in measurement and data processing. It is necessary to analyze these errors and provide solutions to improve the effectiveness of the system [16].

3.2.1. Voltage and Current Sensors. Hall current and voltage sensors are used in this study. Without considering the effect of temperature, the output voltage $\left(U_{V}\right)$ and current $\left(U_{I}\right)$ of Hall sensors are

$$
\begin{aligned}
& U_{V}=\alpha V, \\
& U_{I}=\beta I,
\end{aligned}
$$

where $V$ and $I$ are the measured voltage and current and $\alpha$ and $\beta$ are constants, respectively.

When temperature is taken into account,

$$
\begin{aligned}
U_{V} & =f(V, T), \\
U_{I} & =g(I, T) .
\end{aligned}
$$

Since $f$ and $g$ are unknown functions, each depending on two variables, two-dimensional regression analysis is used to determine the relationship between the measured parameters and sensor outputs. Then the coefficients of the regression equation are calculated using the least square method.

The two-dimensional regression equation is established based on (16):

$$
I=\bar{g}\left(U_{I}, T\right) .
$$


TABLE 1: Data collected by experimental system.

\begin{tabular}{|c|c|c|c|c|c|c|c|c|}
\hline \multirow[t]{2}{*}{ Branch } & \multicolumn{2}{|c|}{1} & \multicolumn{2}{|c|}{2} & \multicolumn{2}{|c|}{3} & \multicolumn{2}{|c|}{4} \\
\hline & $V_{11}$ & $V_{12}$ & $V_{21}$ & $V_{22}$ & $V_{31}$ & $V_{32}$ & $V_{41}$ & $V_{42}$ \\
\hline \multirow[t]{2}{*}{ Voltage $(V)$} & 1.559 & 2.454 & 2.072 & 2.086 & 2.060 & 2.074 & 1.876 & 2.190 \\
\hline & \multicolumn{2}{|c|}{$I_{1}$} & \multicolumn{2}{|c|}{$I_{2}$} & \multicolumn{2}{|c|}{$I_{3}$} & \multicolumn{2}{|c|}{$I_{4}$} \\
\hline \multirow[t]{2}{*}{ Current $(A)$} & \multicolumn{2}{|c|}{0.041} & \multicolumn{2}{|c|}{0.128} & \multicolumn{2}{|c|}{0.121} & \multicolumn{2}{|c|}{0.081} \\
\hline & \multicolumn{2}{|c|}{$T_{e 1}$} & \multicolumn{2}{|c|}{$T_{e 2}$} & \multicolumn{2}{|c|}{$T_{e 3}$} & \multicolumn{2}{|c|}{$T_{e 4}$} \\
\hline \multirow[t]{2}{*}{ Environmental temperature $\left({ }^{\circ} \mathrm{C}\right)$} & \multicolumn{2}{|c|}{24.2} & \multicolumn{2}{|c|}{24.2} & \multicolumn{2}{|c|}{24.2} & \multicolumn{2}{|c|}{24.0} \\
\hline & \multicolumn{2}{|c|}{$T_{p 1}$} & \multicolumn{2}{|c|}{$T_{p 2}$} & \multicolumn{2}{|c|}{$T_{p 3}$} & \multicolumn{2}{|c|}{$T_{p 4}$} \\
\hline \multirow[t]{2}{*}{ Panel temperature $\left({ }^{\circ} \mathrm{C}\right)$} & \multicolumn{2}{|c|}{38.1} & \multicolumn{2}{|c|}{37.4} & \multicolumn{2}{|c|}{37.5} & \multicolumn{2}{|c|}{31.9} \\
\hline & \multicolumn{2}{|c|}{$G_{1}$} & \multicolumn{2}{|c|}{$G_{2}$} & \multicolumn{2}{|c|}{$G_{3}$} & \multicolumn{2}{|c|}{$G_{4}$} \\
\hline Solar irradiance $\left(\mathrm{W} / \mathrm{m}^{2}\right)$ & \multicolumn{2}{|c|}{760} & \multicolumn{2}{|c|}{762} & & & & \\
\hline
\end{tabular}

TABLE 2: Preference of different decision makers.

\begin{tabular}{lccccc}
\hline \multirow{2}{*}{ Decision makers } & \multicolumn{5}{c}{ Preference } \\
& VL & $L$ & $M$ & $H$ & VH \\
\hline$d_{V}$ & 0.00 & 0.00 & 0.00 & 0.00 & 1.00 \\
$d_{I}$ & 0.00 & 0.00 & 0.00 & 0.65 & 0.35 \\
$d_{\mathrm{TE}}$ & 0.20 & 0.20 & 0.20 & 0.20 & 0.20 \\
$d_{\mathrm{TP}}$ & 0.00 & 0.00 & 0.00 & 0.80 & 0.20 \\
$d_{G}$ & 0.20 & 0.20 & 0.20 & 0.20 & 0.20 \\
\hline
\end{tabular}

TABLE 3: Ranks of preference.

\begin{tabular}{lccccc}
\hline \multirow{2}{*}{ Decision makers } & \multicolumn{5}{c}{ Ranks } \\
& VL & $L$ & $M$ & $H$ & VH \\
\hline$d_{V}$ & 5 & 4 & 3 & 2 & 1 \\
$d_{I}$ & 5 & 4 & 3 & 1 & 2 \\
$d_{\mathrm{TE}}$ & 5 & 4 & 3 & 2 & 1 \\
$d_{\mathrm{TP}}$ & 5 & 4 & 3 & 1 & 2 \\
$d_{G}$ & 5 & 4 & 3 & 2 & 1 \\
\hline
\end{tabular}

TABLE 4: Weighted generalized distance.

\begin{tabular}{lccccc}
\hline$Q_{i j}$ & $d_{V}$ & $d_{I}$ & $d_{\mathrm{TE}}$ & $d_{\mathrm{TP}}$ & $d_{G}$ \\
\hline$d_{V}$ & 0.00 & 0.21 & 0.21 & 0.25 & 0.21 \\
$d_{I}$ & 0.21 & 0.00 & 0.18 & 0.05 & 0.18 \\
$d_{\mathrm{TE}}$ & 0.21 & 0.18 & 0.00 & 0.19 & 0.00 \\
$d_{\mathrm{TP}}$ & 0.25 & 0.05 & 0.19 & 0.00 & 0.19 \\
$d_{G}$ & 0.21 & 0.18 & 0.00 & 0.19 & 0.00 \\
\hline
\end{tabular}

TABLE 5: Software evaluation indexes.

\begin{tabular}{lcc}
\hline Decision makers & IAI & IDI \\
\hline$d_{V}$ & 0.00 & 0.00 \\
$d_{I}$ & 0.25 & 0.00 \\
$d_{\mathrm{TE}}$ & 0.25 & 0.00 \\
$d_{\mathrm{TP}}$ & 0.25 & 0.00 \\
$d_{G}$ & 0.25 & 0.00 \\
Combined index & 0.20 & 0.00 \\
\hline
\end{tabular}

It can be expressed as follows:

$$
I=a_{0}+a_{1} U_{I}+a_{2} T+a_{3} U_{I}^{2}+a_{4} U_{I} T+a_{5} T^{2}+\varepsilon,
$$

where $I$ is the corrected current, $a_{0} \sim a_{5}$ are constants that are considered as key factors for $I$, and $\varepsilon$ is infinitesimal.

An error $e$ exists between $I\left(U_{I}, T\right)$ and calibration value $I_{k}$ with a variance of

$$
e^{2}=\left[I_{k}-I\left(U_{I}, T\right)\right]^{2}
$$

At last, $a_{0} \sim a_{5}$ can be estimated by the least square method that makes $e^{2}$ a minimum.

3.2.2. Temperature Measurement. Figure 5 shows a general model of the PV cell, which can be expressed as

$$
I=I_{\mathrm{ph}}-I_{\mathrm{st}}\left\{\exp \left[\frac{q\left(U+\mathrm{IR}_{s}\right)}{n k t}\right]-1\right\}-\frac{U+\mathrm{IR}_{s}}{R_{\mathrm{sh}}},
$$

where $I_{\mathrm{ph}}$ is the photons-generated current due to sunlight, $I_{\text {st }}$ is the diode reverse saturation current, $q$ is an electron charge $\left(1.6 * 10^{-9} \mathrm{C}\right), k$ is Boltzmann's constant $(=1.38 *$ $\left.10^{-23} \mathrm{~J} / \mathrm{K}\right), t$ is working temperature of the cell in Kelvin, $n$ is the ideality factor, $R_{s}$ is the series resistance, and $R_{\mathrm{sh}}$ is the parallel resistance.

It shows that the ambient temperature can affect PV panel temperature, which in turn can affect the output current $I$ and voltage $U$. Therefore, temperature is an important factor contributing to PV panel failure, and the accuracy of temperature measurements has a direct effect on the overall precision of the system. It is thus necessary to compensate the temperature measured by DS18B20, which is known to be vulnerable to thermal noise of internal semiconductor. The error increases linearly with temperature. We partitioned the temperature into different ranges and then calculated the correction coefficient by using a more accurate temperature sensor.

Let the linear error model of DS18B20 be $T=H \times T_{s}+$ $W$, where $T$ is measured by DS18B20, $T_{s}$ is measured by a more accurate sensor and represents the actual temperature at a certain moment, $H$ is a linear correction coefficient that varies with temperature, and $W$ is an error compensation 


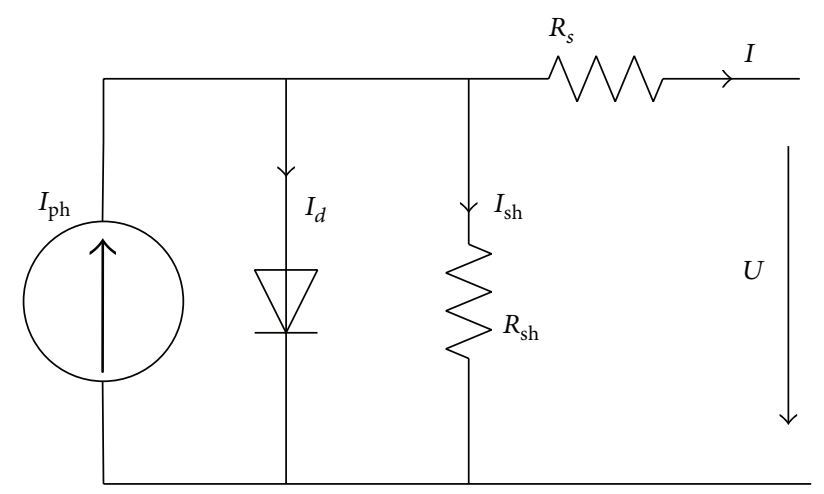

FIgURE 5: Circuit model of solar cell.

parameter. $H$ and $W$ are estimated by observing the temperature for $M$ times:

$$
T_{i}=H \times T_{s i}+W+v_{i} \quad(i=1 \sim M),
$$

where $v_{i}$ is the random error with zero mean in each observation.

Temperature is measured, most probably, under different conditions, thus providing more accurate results in some experiments and less accurate ones in others. In this study, the weighted least squares method is used with a weight matrix of $\bar{W}=\operatorname{diag}\left[w_{1}, w_{2}, \ldots, w_{M}\right]$, where $w_{i}$ is the weight of No.i observation.

Then (21) becomes

$$
T_{i}=H \times \bar{W} \times T_{S i}+W+v_{i} \quad(i=1 \sim M) .
$$

According to the least square method,

$$
\begin{aligned}
{\left[\begin{array}{c}
H \\
W
\end{array}\right]=} & {\left.\left[\begin{array}{cccc}
\frac{T_{S 1}}{w_{1}} & \frac{T_{S 2}}{w_{2}} & \cdots & \frac{T_{S M}}{w_{M}} \\
1 & 1 & \cdots & 1
\end{array}\right] \times\left[\begin{array}{cc}
\frac{T_{S 1}}{w_{1}} & 1 \\
\frac{T_{S 2}}{w_{2}} & 1 \\
\vdots & \vdots \\
\frac{T_{S M}}{w_{M}} & 1
\end{array}\right]\right]^{-1} } \\
& \times\left[\begin{array}{cccc}
\frac{T_{S 1}}{w_{1}} & \frac{T_{S 2}}{w_{2}} & \cdots & \frac{T_{S M}}{w_{M}} \\
1 & 1 & \cdots & 1
\end{array}\right] \times\left[\begin{array}{c}
T_{1} \\
T_{2} \\
\vdots \\
T_{M}
\end{array}\right]
\end{aligned}
$$

It follows from (23) that $H$ for different temperature ranges can be obtained from the temperature measured by DS18B20 and the accurate sensor. Then $H$ values will be stored in microprocessor and used to calculate the temperature.

3.2.3. Solar Irradiance Measurement. It shows in (20) that the output of solar cells depends to a great extent on $I_{\mathrm{ph}}$ determined by the solar irradiance. Therefore, the measurement of solar irradiance will be considered. In this study, it is measured by TSL230B, in which sun light is converted

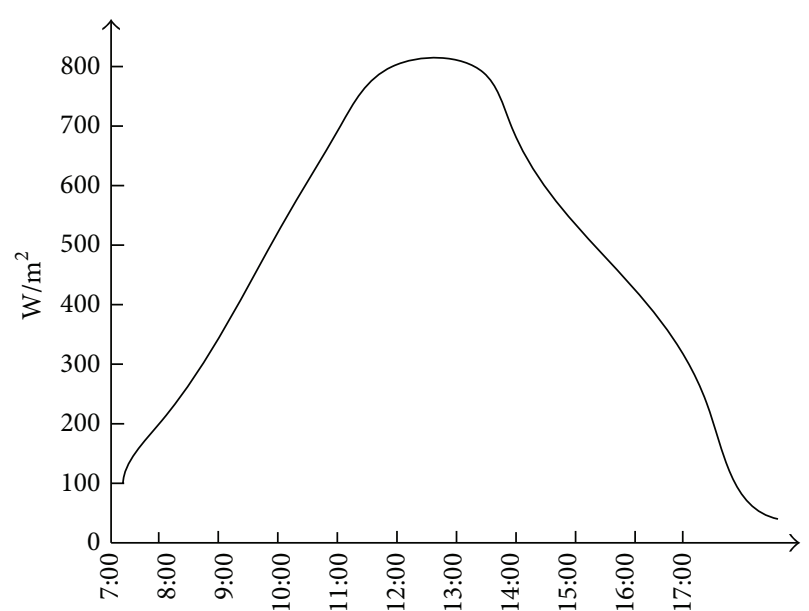

(h)

FIgure 6: Typical solar irradiance curve in north China.

to current by polycrystalline silicon photoelectric diode and then to frequency signals by current-frequency converter. Figure 6 shows a typical solar irradiance curve in north China. It shows that the solar irradiance ranges from about 100 to $800 \mathrm{~W} / \mathrm{m}^{2}$; thus the working time for TSL230B would be very long and its stability would be greatly affected by temperature. It thus points to a need to compensate temperature drift.

Without considering the temperature drift, the relationship between measured solar irradiance $G$ and output frequency $f$ is linear:

$$
G=a f+b,
$$

where $a$ and $b$ are linear coefficients.

When temperature drift is considered,

$$
\bar{G}=a f+b+h(t) .
$$

$H(t)$ is an unknown function that can be expanded by Taylor's formula, and $h(t)$ can be approximated by a polynomial whose coefficients are derivative values:

$$
h(t)=a_{n} t^{n}+a_{n-1} t^{n-1}+\cdots+a_{1} t+a_{0},
$$

where $a_{i}(i=0,1,2, \ldots, n)$ is a parameter.

$\bar{G}$ is measured by a precise handheld irradiance meter under different temperatures $t$, and the frequency $f$ is measured by TSL230B. A polynomial curve is fitted using the Polyfit function in Matlab, so that $a_{i}$ can be obtained. Although a higher degree of fitting appears to be theoretically appealing as it implies a better fitted model, it will pose a high demand on CPU. The daily temperature varies in a parabola manner. In this study, the fitting degree of 3 can completely meet the requirements.

3.2.4. Compared with Other Methods. There have been very few methods on PV array faults diagnosis in practice. It is difficult to determine the location of fault rapidly for SP 
connection structure, when one solar panel fails. The method on infrared images has low accuracy and needs expensive price. The method used in [7] needs large number of voltage sensors and current sensors, which increases the cost of the system. The new sensor-embedded method proposed in this study needs much fewer sensors, which decreases the cost of the whole system. Besides this, if one solar panel fails, fault will be confined to the two adjacent panels rapidly. The accuracy is also improved.

\section{Conclusions}

In this study, a new fault diagnosis method is proposed for PV arrays with SP connection, which is with practical application value, which can minimize the number of sensors needed, decrease the cost of the whole system, and improve the accuracy and anti-interference ability with the introduction of fuzzy group decision-making theory. It makes good use of all relevant information, including voltage, current, environmental temperature, panel temperature, and solar illumination, thereby resulting in a more accurate diagnosis of PV array faults. In addition, errors that cause diagnosis deviation are analyzed, and solutions are suggested to further improve the precision of diagnosis.

\section{Conflict of Interests}

The authors declare that there is no conflict of interests regarding the publication of this paper.

\section{Acknowledgments}

This work is supported financially by Tianjin Municipal Science and Technology Commission under Project no. 09ZCGYGX01100 and by the National Natural Science Fund of China under Project no. 61374122.

\section{References}

[1] A. M. Bazzi, K. A. Kim, B. B. Johnson, P. T. Krein, and A. Dominguez-Garcia, "Fault impacts on solar power unit reliability," in Proceedings of the 26th Annual IEEE Applied Power Electronics Conference and Exposition (APEC '11), pp. 1223-1231, Fort Worth, Tex, USA, March 2011.

[2] F. Ancuta and C. Cepisca, "Fault analysis possibilities for PV panels," in Proceedings of the 3rd International Youth Conference on Energetics (IYCE '11), pp. 1-5, Leiria, Portugal, July 2011.

[3] A. Colli, "Extending performance and evaluating risks of PV systems failure using a fault tree and event tree approach: analysis of the possible application," in Proceedings of the 38th IEEE Photovoltaic Specialists Conference (PVSC '12), pp. 29222926, Austin, Tex, USA, June 2012.

[4] H. Braun, S. T. Buddha, V. Krishnan et al., "Signal processing for fault detection in photovoltaic arrays," in Proceedings of the IEEE International Conference on Acoustics, Speech, and Signal Processing (ICASSP '12), pp. 1681-1684, March 2012.

[5] T. Takashima, J. Yamaguchi, K. Otani, T. Oozeki, K. Kato, and M. Ishida, "Experimental studies of fault location in PV module strings," Solar Energy Materials and Solar Cells, vol. 93, no. 6-7, pp. 1079-1082, 2009.
[6] T. Takashima, K. Otani, K. Sakuta et al., "Electrical detection and specification of failed modules in PV array," in Proceddings of the 3rd World Conference on Photovoltaic Energy Conversion, vol. 3, pp. 2276-2279, May 2003.

[7] Z. Cheng, D. Zhong, B. Li, and Y. Liu, "Research on fault detection of PV array based on data fusion and fuzzy mathematics," in Proceedings of the Asia-Pacific Power and Energy Engineering Conference (APPEEC '11), pp. 1-4, Wuhan, China, March 2011.

[8] D. Nguyen and B. Lehman, "An adaptive solar photovoltaic array using model-based reconfiguration algorithm," IEEE Transactions on Industrial Electronics, vol. 55, no. 7, pp. 26442654, 2008.

[9] Y. Hirata, S. Noro, T. Aoki, and S. Miyazawa, "Diagnosis photovoltaic failure by simple function method to acquire I-V curve of photovoltaic modules string," in Proceedings of the 38th IEEE Photovoltaic Specialists Conference (PVSC '12), pp. 1340-1343, June 2012.

[10] D. Chenvidhya, K. Kirtikara, and C. Jivacate, "PV module dynamic impedance and its voltage and frequency dependencies," Solar Energy Materials and Solar Cells, vol. 86, no. 2, pp. 243-251, 2005.

[11] H. Braun, S. T. Buddha, V. Krishnan et al., "Signal processing for fault detection in photovoltaic arrays," in Proceeding of the IEEE International Conference on Acoustics, Speech, and Signal Processing (ICASSP '12), pp. 1681-1684, Kyoto, Japan, March 2012.

[12] X.-Q. Liu, X. Chen, and H. Zhang, "A multi-character group decision-making method and application based on group ideal solution," Journal of Shenyang Institute of Aeronautical Engineering, vol. 24, no. 2, pp. 38-41, 2007 (Chinese).

[13] Y. He, F. Chu, and B. Zhong, "A study on group decision-making based fault multi-symptom-domain consensus diagnosis," Reliability Engineering and System Safety, vol. 74, no. 1, pp. 43-52, 2001.

[14] Y. Lai, X. Li, Y. Xiong, P. Du, and B. Lu, "Intelligent instrument fault diagnosis expert system based on fuzzy group decisionmaking," Chinese Journal of Scientific Instrument, vol. 29, no. 1, pp. 206-211, 2008 (Chinese).

[15] J. Wang and J. Ren, "Approach to group decision-making with different forms of preference information," Systems Engineering and Electronics, vol. 27, no. 12, pp. 2057-2060, 2005.

[16] J. Jiang, Y. Chen, and D. Tang, “TOPSIS with belief structure for group belief multiple criteria decision making," International Journal of Automation and Computing, vol. 7, no. 3, pp. 359-364, 2010.

[17] G. Yan, C. Liu, and Z. Shao, "Analysis of influencing factors for the grey multi-attribute group decision making," in Proceedings of the IEEE International Conference on Grey Systems and Intelligent Services (GSIS '09), vol. 10, pp. 1081-1086, Nanjing, China, November 2009.

[18] Y. Liu, "Design of a new moisture sensor with auto temperature compensation," Journal of Zhejiang University, vol. 33, pp. 427431, 1999.

[19] Y. Liu, Z. Pang, and Z. Cheng, "Research on an adaptive solar photovoltaic array using shading degree model-based reconfiguration algorithm," in Proceedings of the Chinese Control and Decision Conference (CCDC '10), pp. 2356-2360, May 2010.

[20] X. G. Wang and W. Liu, "A fuzzy fault diagnosis scheme with application," in Proceedings of the Joint 9th IFSA World Congress and 20th NAFIPS International Conference, vol. 3, pp. 14891493, Vancouver, Canada, July 2001. 
[21] G. Yan, C. Liu, and Z. Shao, "Analysis of influencing factors for the grey multi-attribute group decision making," in Proceedings of the IEEE International Conference on Grey Systems and Intelligent Services (GSIS '09), pp. 1081-1086, Nanjing, China, November 2009. 


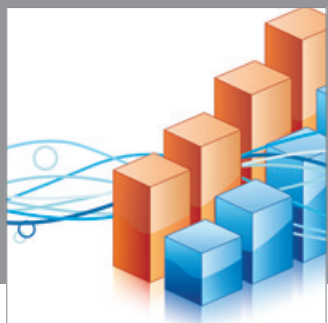

Advances in

Operations Research

mansans

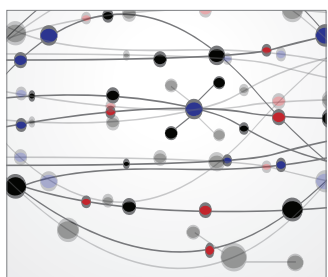

The Scientific World Journal
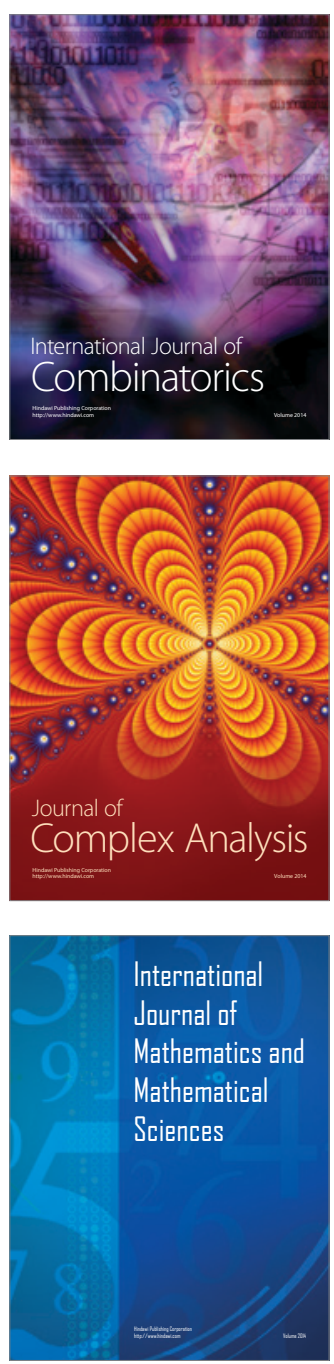
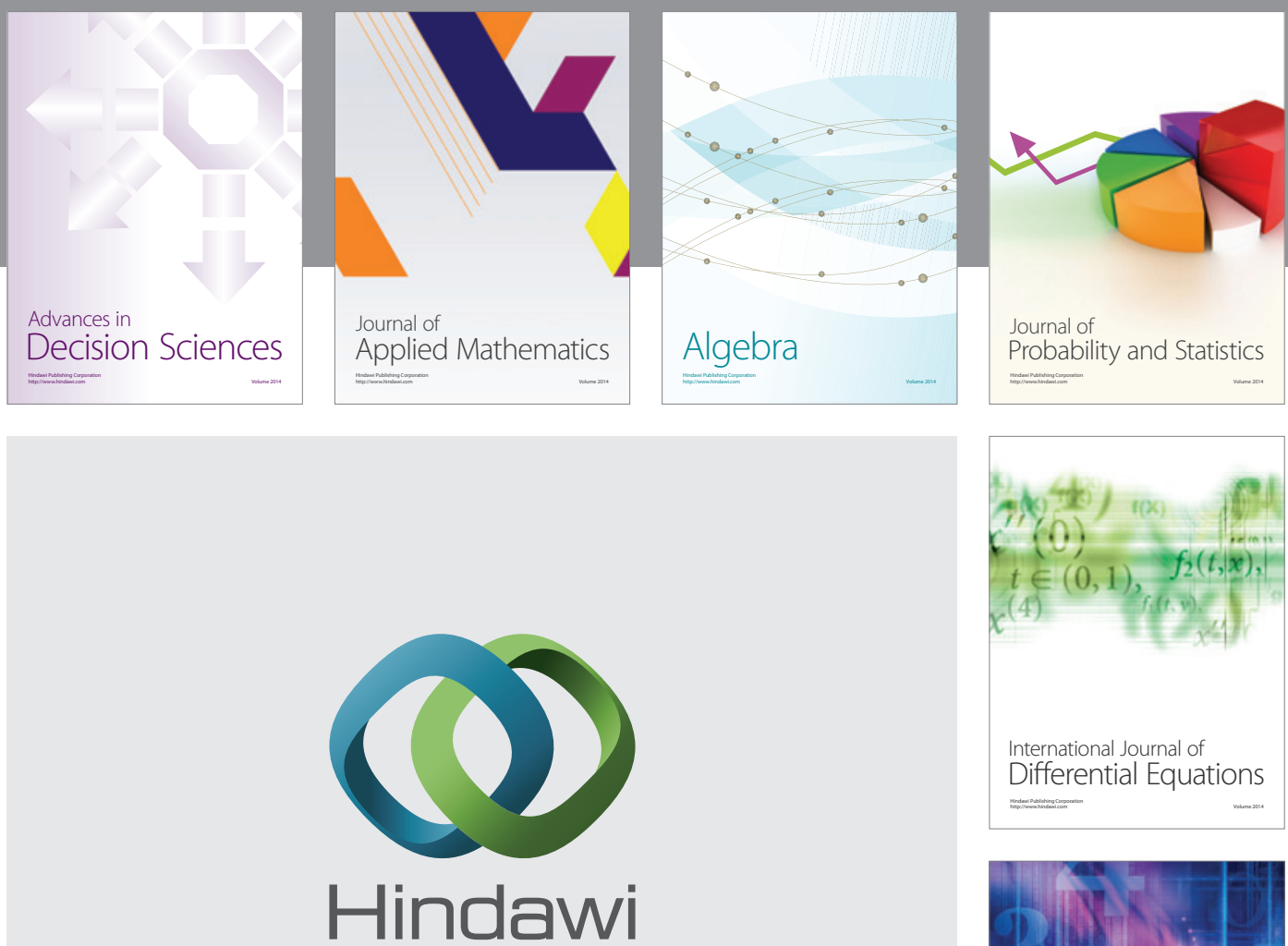

Submit your manuscripts at http://www.hindawi.com
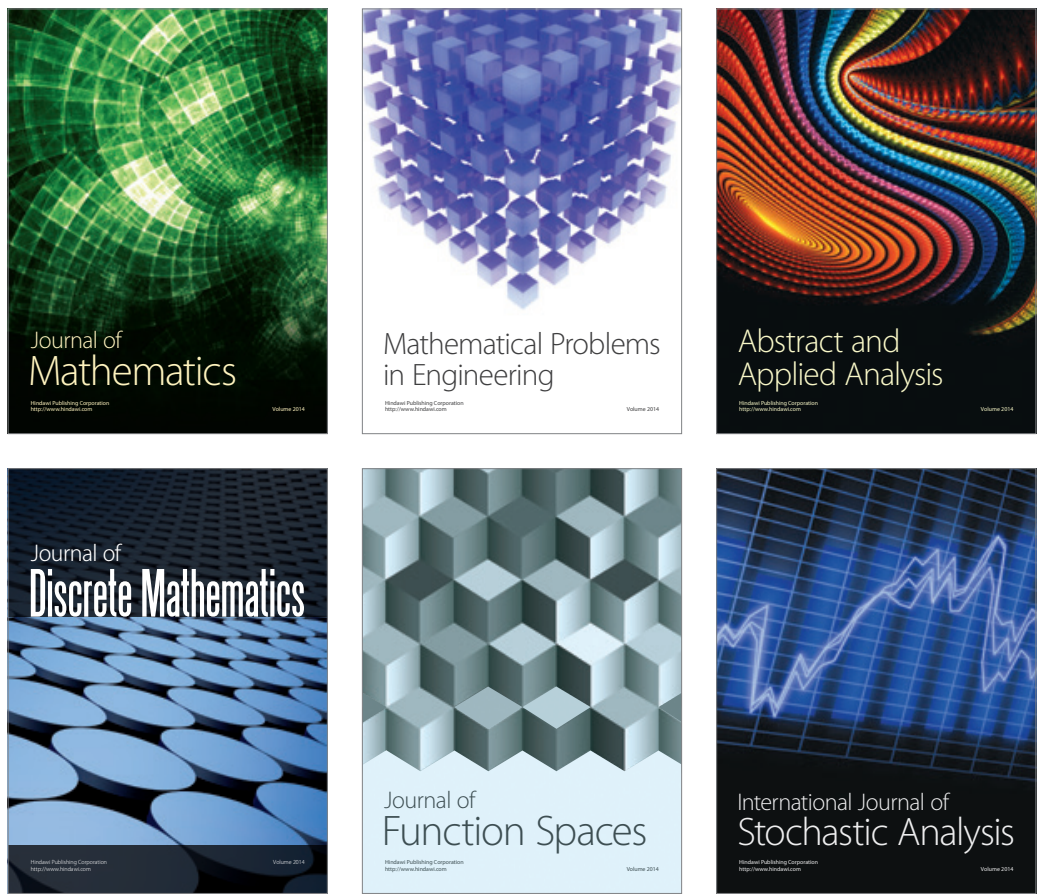

Journal of

Function Spaces

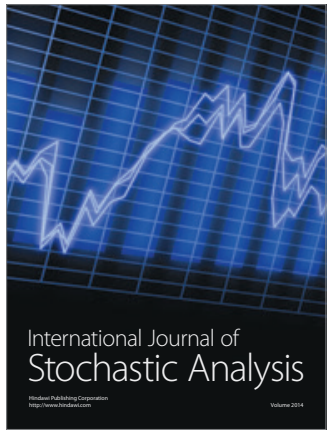

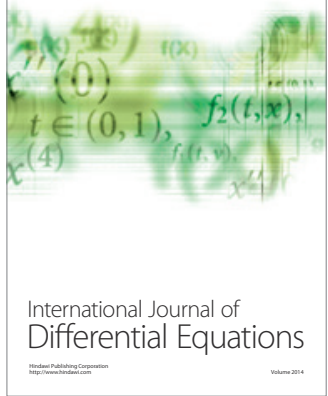
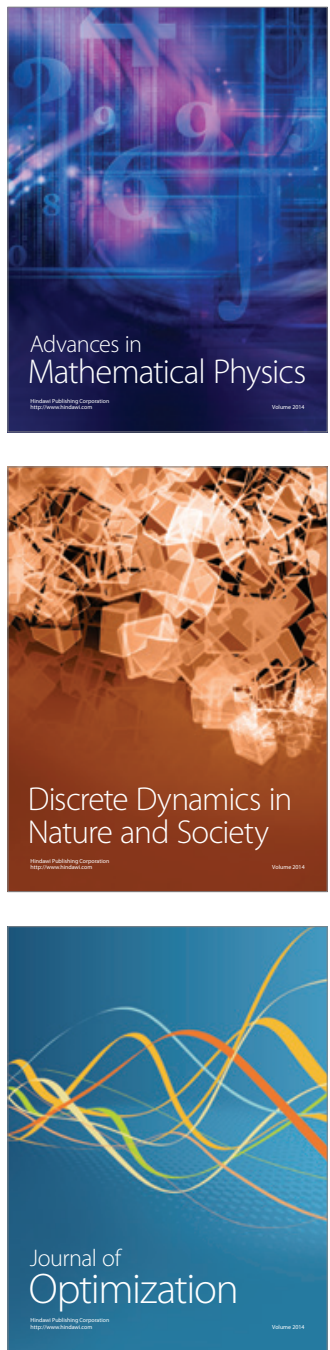